OPEN ACCESS

Check for updates

${ }^{1}$ Department of Epidemiology and Biostatistics, School of

Public Health, Tongji Medical College, Huazhong University of Science and Technology, Wuhan, 430030, China

${ }^{2}$ Ministry of Education Key Laboratory of Environment and Health, and State Key

Laboratory of Environmental Health (Incubating), School of Public Health, Tongji Medical College, Huazhong University of Science and Technology, Wuhan, 430030, China

Correspondence to: An Pan panan@hust.edu.cn

(ORCID 0000-0002-1089-7945)

Additional material is published online only. To view please visit the journal online.

Cite this as: BMJ 2019;367:15584 http://dx.doi.org/10.1136/bmj.15584

Accepted: 29 August 2019

\title{
Weight change across adulthood in relation to all cause and cause specific mortality: prospective cohort study
}

\author{
Chen Chen, ${ }^{1,2}$ Yi Ye, ${ }^{1,2}$ Yanbo Zhang, ${ }^{1,2}$ Xiong-Fei Pan, ${ }^{1,2}$ An Pan ${ }^{1,2}$
}

\author{
ABSTRACT \\ OBJECTIVE \\ To investigate the association between weight \\ changes across adulthood and mortality. \\ DESIGN \\ Prospective cohort study. \\ SETTING \\ US National Health and Nutrition Examination Survey \\ (NHANES) 1988-94 and 1999-2014.
}

\section{PARTICIPANTS}

36051 people aged 40 years or over with measured body weight and height at baseline and recalled weight at young adulthood ( 25 years old) and middle adulthood (10 years before baseline).

\section{MAIN OUTCOME MEASURES}

All cause and cause specific mortality from baseline until 31 December 2015.

\section{RESULTS}

During a mean follow-up of 12.3 years, 10500 deaths occurred. Compared with participants who remained at normal weight, those moving from the non-obese to obese category between young and middle adulthood had a $22 \%$ (hazard ratio $1.22,95 \%$ confidence interval 1.11 to 1.33$)$ and $49 \%(1.49,1.21$ to 1.83$)$ higher risk of all cause mortality and heart disease mortality, respectively. Changing from obese to non-obese body mass index over this period was not significantly associated with mortality risk. An obese to non-obese weight change pattern from middle to late adulthood was associated with increased risk

\section{WHAT IS ALREADY KNOWN ON THIS TOPIC}

High body mass index in adulthood is associated with higher risk of premature death

Scientific evidence on weight change from young to late adulthood or from middle to late adulthood in relation to risk of mortality is not entirely consistent Little is known about associations between weight change across adulthood, especially from young to middle adulthood, and all cause and cause specific mortality

\section{WHAT THIS STUDY ADDS}

Weight gain from young to middle adulthood was associated with increased risk of mortality from all causes and heart diseases in US adults

Weight loss over this period was not significantly related to mortality, and people who remained obese had the highest risk of mortality

Across adulthood, the association between weight gain and mortality attenuated with increasing age, whereas that with weight loss from middle to late adulthood became stronger and significant

The results highlight the importance of maintaining normal weight across adulthood, especially preventing weight gain in early adulthood, for preventing premature deaths in later life of all cause mortality $(1.30,1.16$ to 1.45$)$ and heart disease mortality $(1.48,1.14$ to 1.92$)$, whereas moving from the non-obese to obese category over this period was not significantly associated with mortality risk. Maintaining obesity across adulthood was consistently associated with increased risk of all cause mortality; the hazard ratio was 1.72 (1.52 to 1.95) from young to middle adulthood, 1.61 (1.41 to 1.84) from young to late adulthood, and 1.20 (1.09 to 1.32) from middle to late adulthood. Maximum overweight had a very modest or null association with mortality across adulthood. No significant associations were found between various weight change patterns and cancer mortality.

\section{CONCLUSIONS}

Stable obesity across adulthood, weight gain from young to middle adulthood, and weight loss from middle to late adulthood were associated with increased risks of mortality. The findings imply that maintaining normal weight across adulthood, especially preventing weight gain in early adulthood, is important for preventing premature deaths in later life.

\section{Introduction}

Obesity is a major global public health problem. The global prevalence of obesity in adults increased from $3 \%$ in 1975 to $11 \%$ in 2016 among men and from $6 \%$ to $15 \%$ among women. In the US, the prevalence of obesity in adults was 36\% for men and 38\% for women in 2016 compared with 11\% for men and 14\% for women in $1975 .^{1}$ The U shaped or J shaped relation between body mass index (BMI) and mortality has been well established. ${ }^{2}{ }^{3}$ However, many previous cohort studies included only a single measurement of BMI, which ignored the dynamic feature of body weight over time. Thus more studies are needed to assess the long term consequences of weight change during certain life periods. A recent meta-analysis of 26 prospective studies in participants aged 40 to 65 years at baseline reported that both weight loss and weight gain from middle adulthood to old age were related to elevated mortality risk. ${ }^{4}$ Another meta-analysis of 17 prospective studies in adults aged 60 years or older reached similar conclusions. ${ }^{5}$ However, adults gain weight more rapidly from young to middle adulthood, and excess adiposity mostly accrues in this period compared with the period from middle to late adulthood when weight begins to stabilise or even decrease. ${ }^{6}$ In addition, preventing weight gain from young to middle adulthood might be more important than promoting weight loss, because achieving long term weight loss and maintaining it are difficult once a person becomes obese. ${ }^{7}$ Evaluating the long term effect of weight change, particularly weight 
gain from young to middle adulthood, on future health is thus important.

Although some previous cohort studies reported that weight gain from young adulthood to either midlife or late adulthood was associated with increased mortality risk, ${ }^{8-11}$ the results were not entirely consistent, with some studies reporting no significant association. ${ }^{12} 13$ Some studies found that weight loss was associated with increased mortality risk, ${ }^{12} 1415$ whereas others did not. ${ }^{1011}$ In addition, some studies had only two measurements of weight (from young adulthood at around 20 years old to the baseline survey), ${ }^{9} 111415$ used the trajectory analysis approach when more than two measures of weight were made, ${ }^{1617}$ or were conducted in specific groups rather than the general population. ${ }^{10}{ }^{13}$ A previous study in the US National Health and Nutrition Examination Survey (NHANES) 1988-2004 evaluated the relations of all cause mortality with weight status at age 25 , at 10 years before baseline, and at the baseline survey among adults with a baseline age of 50-74 years. ${ }^{18}$ However, this study focused on the statistical modelling of obesity history and suggested that a duration model performed well in predicting mortality.

Using updated data from NHANES 1988-2014, we aimed to examine the relations of weight changes from young adulthood (age 25 years) to midlife (mean age 47 years) and to late adulthood (mean age 57 years) with all cause and cause specific mortality.

\section{Methods}

Study population

Details of NHANES have been described elsewhere. ${ }^{19}$ Briefly, NHANES used a complex, multistage, probability sampling method to collect nationally representative health related data on the US population. It was conducted periodically before 1999 and on a continuous basis thereafter. Data were obtained by inperson interview, mobile physical examination, and laboratory tests.

In the analysis reported here, we included only participants aged 40 or over at the baseline surveythat is, 40052 participants from the NHANES III (198894) and continuous NHANES (1999-2014) datasets, given that death mostly occurred in older adults. We excluded participants who had a household interview but did not participate in the physical examination $(\mathrm{n}=3198)$, those without baseline BMI $(\mathrm{n}=761)$, and those without mortality data $(n=42)$. Therefore, a total of 36051 participants remained in our cohort for analysis (supplementary figure A).

\section{Assessments of weight change and covariates}

Data on weight at age 25 years and at 10 years before the baseline NHANES survey were recalled at the baseline surveys. Baseline weight and height were measured during mobile physical examination. BMI at age 25 years $\left(\mathrm{BMI}_{25}\right)$, at 10 years before baseline survey (BMI $_{10 \text { prior; }}$; mean age 47 (range 30-80, interquartile range 36-56) years) and at baseline survey $\left(\mathrm{BMI}_{\text {baseline; }}\right.$; mean age 57 (range 40-90, interquartile range 46-
66 years) were calculated as weight (kg) divided by the square of height $\left(\mathrm{m}^{2}\right)$. We categorised each of the three BMI variables into three groups: underweight and normal weight $(<25)$, overweight (25.0-29.9), and obese $(\geq 30.0) .^{20}$ Information on covariates was available through baseline questionnaires, including age, sex, race/ethnicity, education level, family income-poverty ratio, marital status, smoking status, drinking status, leisure time physical activity level, dietary intake data by one or two 24 hour dietary recalls, family history of diabetes or heart attack (because those were consistently collected in various survey cycles), self reported health status, and self reported baseline history of diabetes, hypertension, cardiovascular disease, chronic obstructive pulmonary disease, and cancer.

We created weight change patterns for three time intervals: $\mathrm{BMI}_{25}$ to $\mathrm{BMI}_{10 \text { prior }}, \mathrm{BMI}_{25}$ to $\mathrm{BMI}_{\text {baseline }}$, and $\mathrm{BMI}_{10 \text { prior }}$ to $\mathrm{BMI}_{\text {baseline }}$. Using BMI at two time points, we defined five weight change patterns for each interval (supplementary table A): stable normal pattern $(<25.0$ at both times), maximum overweight pattern (25.0-29.9 at either time but not $\geq 30.0$ at the other time), obese to non-obese pattern $(\geq 30.0$ at younger age and $<30.0$ later), non-obese to obese pattern $(<30.0$ at younger age and $\geq 30$.0 later), and stable obesity ( $\geq 30.0$ at both times). The method has been described in detail in a previous publication using NHANES data. ${ }^{21}$ On the basis of a recent study by Zheng et al, ${ }^{11}$ we also classified absolute weight change in each time interval into five groups: weight loss of at least $2.5 \mathrm{~kg}$, weight change within 2.5 $\mathrm{kg}$ (reference group), weight gain of at least $2.5 \mathrm{~kg}$ but less than $10.0 \mathrm{~kg}$, weight gain of at least $10 \mathrm{~kg}$ but less than $20.0 \mathrm{~kg}$, and weight gain of at least $20.0 \mathrm{~kg}$.

\section{Ascertainment of deaths}

We ascertained mortality status by probabilistic matching to the National Death Index through 31 December 2015 using a unique study identifier. Further details of the matching method are available from the National Center for Health Statistics. ${ }^{22}$

We classified causes of deaths according to the codes of ICD-10 (international statistical classification of diseases, 10th revision). Primary outcomes for our study were mortality from all causes, heart diseases (codes I00-I09, I11, I13, and I20-I51), cancer (codes C00-C97), and other causes. Data on mortality from stroke (I60-I69) were available only until 31 December 2011 in the 2011 version of the National Death Index matched mortality dataset.

\section{Statistical analysis}

We accounted for complex survey design factors for NHANES, including sample weights, clustering, and stratification, whenever feasible as specified in the instructions for using NHANES data. ${ }^{19}$ We compared baseline characteristics by weight change patterns in the three intervals by using the Rao-Scott $\chi^{2}$ test for categorical variables and analysis of variance adjusted for sampling weights for continuous variables. ${ }^{23}$ We calculated Pearson correlation coefficients between 
BMI at the three time points and weight change in the three intervals. We defined baseline as the time when participants had their physical examinations. We counted person years from baseline to the date of death, loss to follow-up, or 31 December 2015, whichever came first. We used Cox proportional hazards models with time in study as the underlying time metric to calculate the hazard ratios and corresponding 95\% confidence intervals for mortality in relation to BMI at each time point and weight change patterns in the three time intervals. We examined the proportional hazards assumption by creating a cross product of follow-up time and weight change patterns. Likelihood ratio tests comparing models with and without this variable were not significant, suggesting no departure from the proportional hazards assumption.

We first examined the relations of BMI at each time point and mortality. In this analysis, we categorised BMI into seven groups-underweight $(<18.5)$, three categories of normal weight (18.5-19.9, 20.0-22.4, and 22.5-24.9), overweight (25.0-29.9), and two categories of obesity (30.0-34.9 and $\geq 35.0$ )-and used 22.5-24.9 as the reference group. ${ }^{24}$ For the main analyses, we examined the associations between the five weight change patterns in the three time intervals and mortality: BMI changes from age 25 years to 10 years before baseline $\left(\mathrm{BMI}_{25}\right.$ to $\left.\mathrm{BMI}_{10 \text { prior }}\right)$, which approximated weight change from young to middle adulthood; BMI changes from 10 years before baseline to baseline $\left(\mathrm{BMI}_{10 \text { prior }}\right.$ to $\left.\mathrm{BMI}_{\text {baseline }}\right)$, which approximated weight change from midlife to late adulthood; and BMI changes from age 25 years to baseline $\left(\mathrm{BMI}_{25}\right.$ to $\mathrm{BMI}_{\text {baseline }}$ ), which approximated weight change during the whole adulthood period. We also investigated the associations between absolute weight change groups in the above three time intervals and mortality risk, as well as the possible non-linear relation by nonparametrically restricted cubic splines. ${ }^{25}$ Participants with missing BMI data at certain time points were excluded from the corresponding analysis.

We adjusted for baseline age (years, continuous), sex, and race/ethnicity (non-Hispanic white, nonHispanic black, Mexican American, and others) in model 1. We further adjusted for education level (less than high school, high school or equivalent, and college or above), family income-poverty ratio level $(0-1.0,1.1-3.0,>3.0),{ }^{26}$ marital status (married, separated including widowed and divorced groups, and never married), smoking status (never, former, and current smoker), drinking status (non-drinker, low to moderate drinker defined as drinking < 1 drink/day in women and $<2$ drinks/day in men, and heavy drinker defined as $\geq 1$ drink/day in women and $\geq 2$ drinks/day in men), ${ }^{27}$ and family history of diabetes or heart attack in model 2. We additionally adjusted for baseline moderate to vigorous leisure time physical activity level $\left(0,1-2\right.$, or $\geq 3$ times/week) ${ }^{28}$ dietary pattern represented by the healthy eating index scores (HEI1995 for NHANES 1988-94 and HEI-2015 for NHANES 1999-2014, both in quarters), ${ }^{29}$ and self reported health status (very good to excellent, good, and poor to fair). In the absolute weight change analyses, we also included baseline height and weight at the beginning of the change period as potential confounders in model 2. Finally, we additionally adjusted for baseline histories of diabetes, hypertension, cardiovascular disease, cancer (excluding skin cancer), and chronic obstructive pulmonary disease, as those comorbidities could be mediators for the association between weight change and mortality. Percentages of missing values of covariates were less than 5\% except for income (7.2\%) and drinking status (5.2\%). We did multiple imputation for all missing values. ${ }^{30}$ We did stratified analyses and potential effect modifications by baseline age $(<60$ and $\geq 60$ years), sex, and smoking status (ever and never smokers). To evaluate the effect on premature death, we examined the relations with deaths occurring before 65 years old, before 70 years old, before 75 years old, and before 80 years old among participants younger than 65 years old at baseline.

We did a series of sensitivity analyses to test the robustness of the results. Firstly, we did a complementary analysis of cardiovascular disease mortality and stroke mortality up to 31 December 2011 among participants from NHANES 1988-2010. Secondly, we removed participants with missing values for covariates and ran complete case analyses. Thirdly, we excluded underweight participants to reduce the influence of underweight on the results. Fourthly, we excluded participants who had follow-up time of less than three years (including those who died within three years of follow-up) or excluded participants with prevalent diabetes, cardiovascular disease, or cancer to minimise potential reverse causation caused by severe illness. Finally, we redefined the weight change patterns by combinations of three BMI categories (underweight and normal weight, overweight, and obesity) in each two time points, which generated a nine category weight change group instead of the five weight change patterns. We used SAS 9.4 for all analyses and considered a two tailed $P$ value of $<0.05$ to be statistically significant.

\section{Patient and public involvement}

No participants were involved in setting the research questions or outcome measures, or in the design and implementation of the study. No plans exist to involve patients in dissemination.

\section{Results}

Baseline characteristics and weight change pattern The correlation coefficients between $\mathrm{BMI}_{25}$, $\mathrm{BMI}_{10 \text { prior}}$, and $\mathrm{BMI}_{\text {baseline }}$ ranged from 0.46 to 0.69 (supplementary table B). On average, participants gained $13.4 \mathrm{~kg}$ weight from age 25 years to baseline, $8.8 \mathrm{~kg}$ from age 25 years to 10 years before baseline, and $4.4 \mathrm{~kg}$ in the 10 year period before baseline (table 1 ; supplementary tables $\mathrm{C}$ and D). From age 25 years to baseline, 26.9\% of the participants moved from non-obesity to obesity and they gained $28.2 \mathrm{~kg}$ on average, whereas only $1.4 \%$ of the participants moved from the obese to non-obese category and they lost $18.6 \mathrm{~kg}$ on average (table 1). 
From age 25 years to 10 years before baseline, $42.3 \%$ of the participants were in the stable normal group and $5.2 \%$ were in the stable obese group (supplementary table C). The corresponding numbers were $26.9 \%$ and $16.3 \%$, respectively, for the 10 year period before baseline (supplementary table D).

Table 1 shows baseline characteristics of study participants across weight change patterns from age 25 years to baseline. Compared with people in the stable normal group, those in the other groups were more likely to be men, non-Hispanic black or Mexican American, less educated, poor, and former smokers and more likely to report a family history of diabetes or heart attack and prevalent diabetes, hypertension, or cardiovascular disease; they were less likely to be heavy drinkers or physically active, to have good diet quality, and to report very good to excellent health status. Participants in the stable obese group tended to be younger, whereas those in the obese to non-obese category and maximum overweight category tended to be older, and those in the non-obese to obese category were not significantly different from the stable normal group. Participants in the obese to non-obese group were more likely to be current smokers, whereas the other three weight change groups were less likely to be current smokers compared with the stable normal group. The distributions of participants' characteristics according to the weight change pattern in the other two time periods are shown in supplementary tables $\mathrm{C}$ and $\mathrm{D}$, and the distribution patterns were generally similar except for some inconsistent patterns in age and smoking status.

\section{Relations of weight and weight change patterns with mortality}

Among 36051 participants, 10500 deaths occurred during a mean follow-up of 12.3 years (358980 person years), including 2287 deaths from heart diseases and 2316 from cancer. When evaluating the weight status at each time point (supplementary table E), we found a linear association between BMI at age 25 years and mortality. Overweight and obesity were significantly associated with increased risks of all cause mortality, whereas underweight showed no association. When the participants got older, the association started to change to a U shape; the association between underweight and mortality became significant and stronger, whereas the association with obesity was substantially attenuated and the association with overweight became null. We found similar results when we excluded participants with prevalent diabetes, cardiovascular disease, or cancer at the specific time point or restricted the analysis to never smokers (supplementary table E).

Table 2 shows the association between weight change patterns in the three periods and mortality risk, using the stable normal group as the reference. As expected, stable obese participants had increased risks of all cause mortality across adulthood, with hazard ratios of 1.72 (95\% confidence interval 1.52 to 1.95 ) from age 25 years to 10 years before baseline, 1.61 (1.41 to 1.84 ) from age 25 years to baseline, and 1.20
(1.09 to 1.32 ) in the 10 year period before baseline. Moving from the non-obese range at age 25 years to the obese range at 10 years before baseline was associated with a $22 \%$ higher risk of all cause mortality (hazard ratio $1.22,1.11$ to 1.33 ), whereas the hazard ratio was 1.01 ( 0.92 to 1.11 ) for the period from age 25 years to baseline and 1.01 ( 0.88 to 1.15 ) in the 10 year period before baseline. The maximum overweight group showed a null association in all three time intervals, with hazard ratios of 1.05 (0.98 to 1.12), 0.92 (0.84 to 1.00), and 0.93 (0.84 to 1.02). Changing from obesity to non-obesity in the 10 year period before baseline was associated with a 30\% higher risk of all cause mortality (hazard ratio 1.30, 1.16 to 1.45 ); the hazard ratio was 1.22 (0.87 to 1.69 ) from age 25 years to 10 years before baseline and 1.13 (0.86 to 1.49) from age 25 years to baseline.

We observed similar patterns of results for mortality from heart disease (table 2) and cardiovascular disease (supplementary table F), but with slightly stronger effect estimates. For example, the hazard ratio for heart disease mortality was 2.43 (1.84 to 3.20) for stable obesity from age 25 years to 10 years before baseline, 2.15 (1.58 to 2.92) from age 25 years to baseline, and 1.44 (1.18 to 1.76 ) in the 10 year period before baseline. Likewise, participants who were non-obese at age 25 years but became obese at 10 years before baseline had a hazard ratio of 1.49 (1.21 to 1.83) for heart disease mortality. In addition, the associations with cardiovascular disease mortality were mainly driven by heart disease mortality rather than stroke mortality (supplementary table F). However, the sample size was much smaller for stroke mortality. We found no significant associations between BMI change and cancer mortality for all weight change groups in each time interval (table 2). For deaths from other causes, the results were generally similar to those for all cause mortality.

Further adjustment for baseline comorbidities attenuated the associations, but most significant associations remained (supplementary table $\mathrm{G}$ ). The results were almost unchanged when we removed participantswithmissingvaluesforcovariates, excluded underweight participants, excluded participants who had less than three years of follow-up time, or excluded participants with prevalent diabetes, cardiovascular disease, or cancer (supplementary table H). Because the maximum overweight group was a heterogeneous group with people in the stable overweight group, overweight to normal group, and normal to overweight group, we further classified participants into the nine category weight change groups (supplementary figure B). Compared with the stable normal group, stable overweight was not significantly associated with mortality risk except for an inverse association in the 10 year period before baseline (hazard ratio 0.89 , 0.80 to 0.98 ); the overweight to normal pattern was consistently associated with higher mortality risk in the three time periods (hazard ratio ranged from 1.21 to 1.28 ), whereas the normal to overweight pattern was associated with lower mortality risk from age 25 years 


\section{Table 1 | Baseline characteristics of study participants in NHANES 1988-2014 according to weight change patterns from age 25 years to baseline*. Values are numbers (percentages) unless stated otherwise}

\section{Weight change patterns from age 25 years to baseline}

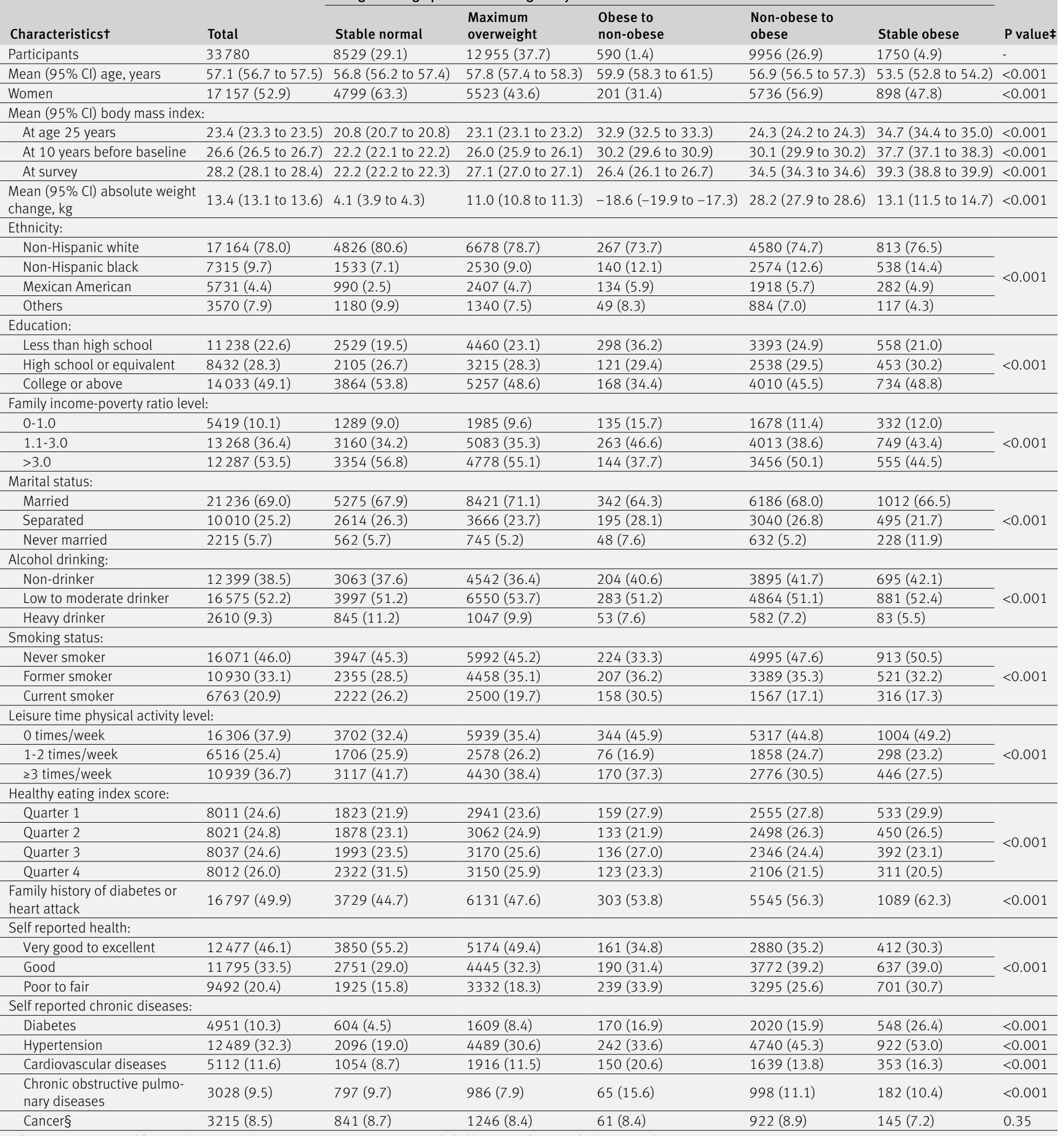

*All estimates accounted for complex survey designs. 2271 participants were excluded because of missing body mass index at age 25 years.

$† 77,2806,319,2196,16,19,1699$, and 16 participants had missing information for baseline education level, family income-poverty ratio, marital status, alcohol drinking, smoking status, leisure time physical activity level, healthy eating index score, and self reported health, respectively.

fFor categorical variables, P value was calculated by Rao-Scott $\mathrm{x}^{2}$ test, which is design adjusted version of Pearson $\mathrm{x}^{2}$ test. For continuous variables, analysis of variance adjusting for sampling weights was used to calculate $P$ value.

$\S S$ in cancer was not included. 


\begin{tabular}{|c|c|c|c|c|c|}
\hline \multirow[b]{2}{*}{ Cause of death } & \multicolumn{5}{|c|}{ Weight change patterns } \\
\hline & Stable normal & Maximum overweight & Obese to non-obese & Non-obese to obese & Stable obese \\
\hline \multicolumn{6}{|c|}{ From age 25 years to 10 years before baseline } \\
\hline \multicolumn{6}{|c|}{ All causes: } \\
\hline No of deaths/person years & $3075 / 132955$ & $3821 / 126939$ & $159 / 3688$ & $1827 / 54259$ & $516 / 16133$ \\
\hline Age adjusted mortality ratet & $26.3(25.4$ to 27.3$)$ & $27.6(26.7$ to 28.5$)$ & $38.2(32.1$ to 44.2$)$ & 30.1 (28.7 to 31.5$)$ & $38.7(35.2$ to 42.1$)$ \\
\hline Model 1 & 1.00 & 1.04 (0.98 to 1.12$)$ & $1.43(1.06$ to 1.92$)$ & $1.30(1.18$ to 1.42$)$ & 1.84 (1.63 to 2.08$)$ \\
\hline Model 2 & 1.00 & $1.07(1.01$ to 1.14$)$ & $1.32(0.95$ to 1.83$)$ & $1.32(1.21$ to 1.45$)$ & $1.90(1.69$ to 2.14$)$ \\
\hline Model 3 & 1.00 & $1.05(0.98$ to 1.12$)$ & $1.22(0.87$ to 1.69$)$ & $1.22(1.11$ to 1.33$)$ & $1.72(1.52$ to 1.95$)$ \\
\hline \multicolumn{6}{|l|}{ Heart diseases: } \\
\hline No (\%) deaths & $569(31.7)$ & $862(41.3)$ & $33(1.1)$ & $409(19.3)$ & $139(6.5)$ \\
\hline Model 1 & 1.00 & $1.18(1.02$ to 1.38$)$ & $1.06(0.67$ to 1.67$)$ & $1.61(1.32$ to 1.96$)$ & 2.68 (2.04 to 3.53$)$ \\
\hline Model 2 & 1.00 & $1.22(1.05$ to 1.41$)$ & 0.94 (0.57 to 1.55$)$ & $1.62(1.32$ to 1.99$)$ & 2.70 (2.07 to 3.53$)$ \\
\hline Model 3 & 1.00 & $1.19(1.02$ to 1.39$)$ & 0.85 (0.49 to 1.48$)$ & 1.49 (1.21 to 1.83$)$ & $2.43(1.84$ to 3.20$)$ \\
\hline \multicolumn{6}{|l|}{ Cancer: } \\
\hline No (\%) deaths & $787(40.1)$ & $868(38.2)$ & $27(1.3)$ & $378(16.8)$ & $90(3.7)$ \\
\hline Model 1 & 1.00 & $0.96(0.83$ to 1.11$)$ & $1.10(0.60$ to 2.02$)$ & $1.20(0.97$ to 1.48$)$ & 1.08 (0.81 to 1.43$)$ \\
\hline Model 2 & 1.00 & $1.01(0.88$ to 1.16$)$ & $1.04(0.56$ to 1.91$)$ & $1.26(1.02$ to 1.56$)$ & 1.17 (0.89 to 1.54$)$ \\
\hline Model 3 & 1.00 & 0.99 (0.86 to 1.15$)$ & 0.97 (0.53 to 1.79$)$ & $1.20(0.96$ to 1.50$)$ & 1.11 (0.85 to 1.47$)$ \\
\hline \multicolumn{6}{|l|}{ Other causes: } \\
\hline No (\%) deaths & $1719(37.5)$ & 2091 (3.9.3) & $99(1.9)$ & $1040(16.2)$ & $287(5.1)$ \\
\hline Model 1 & 1.00 & 1.04 (0.96 to 1.13$)$ & 1.71 (1.14 to 2.55$)$ & 1.25 (1.11 to 1.40$)$ & 1.97 (1.61 to 2.41$)$ \\
\hline Model 2 & 1.00 & $1.06(0.97$ to 1.15$)$ & $1.62(1.08$ to 2.43$)$ & $1.26(1.12$ to 1.41$)$ & 2.01 (1.65 to 2.46$)$ \\
\hline Model 3 & 1.00 & $1.03(0.94$ to 1.12$)$ & $1.49(0.98$ to 2.24$)$ & 1.14 (1.01 to 1.29$)$ & $1.79(1.46$ to 2.20$)$ \\
\hline \multicolumn{6}{|l|}{ From age 25 years to baseline } \\
\hline \multicolumn{6}{|l|}{ All causes: } \\
\hline No of deaths/person years & $2677 / 87892$ & $3915 / 131968$ & $264 / 4923$ & $2302 / 98464$ & $423 / 15156$ \\
\hline Age adjusted mortality rate $\dagger$ & 29.1 (28.0 to 30.2$)$ & $27.8(26.9$ to 28.7$)$ & $45.2(39.5$ to 50.8$)$ & $26.0(24.9$ to 27.2$)$ & $36.3(32.6$ to 40.1$)$ \\
\hline Model 1 & 1.00 & $0.91(0.84$ to 0.99$)$ & $1.38(1.10$ to 1.73$)$ & $1.08(0.99$ to 1.17$)$ & 1.68 (1.48 to 1.92$)$ \\
\hline Model 2 & 1.00 & 0.94 (0.86 to 1.02$)$ & $1.24(0.95$ to 1.60$)$ & $1.10(1.01$ to 1.20$)$ & 1.79 (1.57 to 2.04$)$ \\
\hline Model 3 & 1.00 & $0.92(0.84$ to 1.00$)$ & $1.13(0.86$ to 1.49$)$ & $1.01(0.92$ to 1.11$)$ & 1.61 (1.41 to 1.84$)$ \\
\hline \multicolumn{6}{|l|}{ Heart diseases: } \\
\hline No (\%) deaths & $534(28.2)$ & 846 (38.9) & $66(1.8)$ & $498(25.3)$ & $110(5.7)$ \\
\hline Model 1 & 1.00 & $0.97(0.82$ to 1.16$)$ & $1.26(0.86$ to 1.84$)$ & $1.22(1.02$ to 1.46$)$ & 2.31 (1.70 to 3.15$)$ \\
\hline Model 2 & 1.00 & 0.99 (0.83 to 1.18$)$ & $1.07(0.71$ to 1.61$)$ & $1.22(1.03$ to 1.45$)$ & 2.39 (1.78 to 3.21$)$ \\
\hline Model 3 & 1.00 & $0.97(0.81$ to 1.16$)$ & $0.97(0.63$ to 1.50$)$ & $1.14(0.95$ to 1.35$)$ & 2.15 (1.58 to 2.92$)$ \\
\hline \multicolumn{6}{|l|}{ Cancer: } \\
\hline No (\%) deaths & $604(30.3)$ & $919(38.9)$ & $47(1.5)$ & $545(25.8)$ & $73(3.4)$ \\
\hline Model 1 & 1.00 & $0.92(0.79$ to 1.07$)$ & 1.04 (0.67 to 1.62$)$ & $1.05(0.92$ to 1.21$)$ & 1.05 (0.75 to 1.48$)$ \\
\hline Model 2 & 1.00 & $0.98(0.83$ to 1.14$)$ & $0.96(0.61$ to 1.49$)$ & $1.12(0.97$ to 1.29$)$ & 1.19 (0.86 to 1.65$)$ \\
\hline Model 3 & 1.00 & $0.96(0.82$ to 1.12$)$ & $0.90(0.58$ to 1.40$)$ & $1.06(0.92$ to 1.22$)$ & 1.12 (0.80 to 1.55$)$ \\
\hline \multicolumn{6}{|l|}{ Other causes: } \\
\hline No (\%) deaths & $1539(31.5)$ & $2150(37.7)$ & $151(2.2)$ & 1259 (23.9) & $240(4.7)$ \\
\hline Model 1 & 1.00 & 0.89 (0.81 to 0.98$)$ & 1.58 (1.21 to 2.08$)$ & 1.05 (0.94 to 1.17$)$ & 1.80 (1.45 to 2.22$)$ \\
\hline Model 2 & 1.00 & $0.91(0.83$ to 1.00$)$ & 1.44 (1.06 to 1.95$)$ & $1.06(0.95$ to 1.19$)$ & 1.89 (1.53 to 2.33$)$ \\
\hline Model 3 & 1.00 & $0.88(0.80$ to 0.98$)$ & $1.30(0.94$ to 1.80$)$ & $0.97(0.85$ to 1.10$)$ & 1.68 (1.35 to 2.08 ) \\
\hline From 10 years before baselin & seline & & & & \\
\hline All causes: & & & & & \\
\hline No of deaths/person years & $2362 / 81771$ & $3924 / 131762$ & $852 / 16428$ & $1137 / 58251$ & $1652 / 56972$ \\
\hline Age adjusted mortality rate $\dagger$ & 28.7 (27.5 to 29.9$)$ & 27.8 (26.9 to 28.7$)$ & $40.5(37.7$ to 43.4$)$ & $25.3(23.7$ to 26.9$)$ & 29.7 (28.2 to 31.2$)$ \\
\hline Model 1 & 1.00 & $0.93(0.85$ to 1.02$)$ & 1.46 (1.27 to 1.68$)$ & 1.07 (0.95 to 1.20$)$ & 1.27 (1.15 to 1.40$)$ \\
\hline Model 2 & 1.00 & $0.96(0.88$ to 1.05$)$ & $1.43(1.27$ to 1.60$)$ & $1.09(0.95$ to 1.25$)$ & 1.32 (1.21 to 1.45 ) \\
\hline Model 3 & 1.00 & 0.93 (0.84 to 1.02$)$ & $1.30(1.16$ to 1.45$)$ & $1.01(0.88$ to 1.15$)$ & 1.20 (1.09 to 1.32 ) \\
\hline Heart diseases: & & & & & \\
\hline No (\%) deaths & $462(24.9)$ & $851(37.4)$ & $207(7.3)$ & $243(11.9)$ & $382(18.5)$ \\
\hline Model 1 & 1.00 & 0.94 (0.79 to 1.13$)$ & 1.70 (1.32 to 2.19$)$ & $1.10(0.89$ to 1.35$)$ & 1.56 (1.27 to 1.92$)$ \\
\hline Model 2 & 1.00 & $0.97(0.81$ to 1.15$)$ & 1.63 (1.27 to 2.10$)$ & 1.11 (0.90 to 1.35$)$ & 1.60 (1.31 to 1.95$)$ \\
\hline Model 3 & 1.00 & $0.93(0.78$ to 1.12$)$ & $1.48(1.14$ to 1.92$)$ & 1.03 (0.84 to 1.26$)$ & 1.44 (1.18 to 1.76$)$ \\
\hline Cancer: & & & & & \\
\hline No (\%) deaths & $567(27.8)$ & $892(38.5)$ & $138(4.8)$ & $273(13.2)$ & $354(15.7)$ \\
\hline Model 1 & 1.00 & 0.91 (0.76 to 1.10$)$ & $1.13(0.85$ to 1.51$)$ & 0.95 (0.75 to 1.21$)$ & 1.14 (0.95 to 1.35$)$ \\
\hline Model 2 & 1.00 & $0.96(0.80$ to 1.15$)$ & 1.14 (0.88 to 1.48$)$ & 1.00 (0.79 to 1.28$)$ & 1.25 (1.04 to 1.49$)$ \\
\hline Model 3 & 1.00 & $0.94(0.78$ to 1.13$)$ & $1.08(0.83$ to 1.39$)$ & 0.95 (0.74 to 1.21$)$ & 1.17 (0.98 to 1.40$)$ \\
\hline Other causes: & & & & & \\
\hline No (\%) deaths & $1333(27.2)$ & 2181 (38.1) & $507(6.3)$ & $621(13.4)$ & $916(15.1)$ \\
\hline Model 1 & 1.00 & 0.93 (0.85 to 1.03$)$ & $1.52(1.26$ to 1.84$)$ & $1.12(0.96$ to 1.31$)$ & 1.23 (1.09 to 1.40$)$ \\
\hline Model 2 & 1.00 & $0.96(0.87$ to 1.06$)$ & $1.47(1.24$ to 1.74$)$ & $1.14(0.96$ to 1.35$)$ & 1.27 (1.12 to 1.44$)$ \\
\hline Model 3 & 1.00 & $0.92(0.83$ to 1.03$)$ & $1.32(1.12$ to 1.56$)$ & $1.04(0.87$ to 1.24$)$ & 1.13 (0.99 to 1.30$)$ \\
\hline
\end{tabular}

${ }^{*}$ All estimates accounted for complex survey designs. Among 36051 participants, a total of 2751, 2271, and 1469 participants were excluded in analyses from age 25 years to 10 years before baseline, age 25 years to baseline, and 10 years before baseline to baseline, respectively, owing to missing values of body mass index at both times. Model 1 was adjusted for baseline age, sex, race/ethnicity. Model 2 was additionally adjusted for education level, family income-poverty ratio level, marital status, drinking and smoking status, and family history of diabetes or heart attack. Model 3 was further adjusted for baseline leisure time physical activity level, healthy eating index scores, and self reported general health.

tMortality rates per 1000 person years, directly standardised to age distribution of entire study population. 
to baseline (hazard ratio $0.88,0.80$ to 0.96$)$ and in the 10 year period before baseline $(0.85,0.75$ to 0.95$)$, but not from age 25 years to 10 years before baseline.

In the stratified analyses, we found significant interactions with baseline age but not with smoking status and sex (fig 1), and the associations were stronger among participants who were less than 60 years old at baseline compared with their counterparts. When stratified by age at death, the associations were similar to our main results (supplementary table I).

When evaluating the absolute weight changes, we identified a J shaped or U shaped association for all cause mortality with weight change across the three time intervals (fig 2). When classified into categories (table 3), the hazard ratios for all cause mortality in the extreme weight gain (weight gain $\geq 20 \mathrm{~kg}$ ) group were 1.19 (1.07 to 1.32 ) from age 25 years to 10 years before baseline, 1.04 (0.94 to 1.15) from age 25 years to baseline, and 1.36 (1.17 to 1.57) in the 10 year period before baseline, compared with the stable weight group (weight change within $2.5 \mathrm{~kg}$ ). Moderate to large weight gain (weight gain $\geq 10 \mathrm{~kg}$ and $<20 \mathrm{~kg}$ ) and small to moderate weight gain (weight gain $\geq 2.5$ $\mathrm{kg}$ and $<10 \mathrm{~kg}$ ) were not significantly associated with all cause mortality in any of the three time intervals. Participants who lost more than $2.5 \mathrm{~kg}$ from age 25 years to baseline had a hazard ratio of 1.19 (1.04 to 1.35) for all cause mortality, with a hazard ratio of 1.26 (1.14 to 1.39 ) in the 10 year period before baseline, whereas the association was not significant from age 25 years to 10 years before baseline (hazard ratio 1.10, 0.94 to 1.28). The non-linear associations (J shaped or $\mathrm{U}$ shaped) between absolute weight change and cause specific mortality were observed in the three time intervals, except that neither a linear nor a non-linear association was found for heart disease mortality with weight change from age 25 years to 10 years before baseline (supplementary figure $\mathrm{C}$ ).

\section{Discussion}

In this large prospective study of nationally representative US adults, the lowest risk of mortality was in stable normal participants, whereas both stable obesity across adulthood and weight gain from young to middle adulthood were associated with increased risk of mortality; in addition, weight loss from middle to late adulthood was significantly related to increased mortality risk. The findings underscore the importance of maintaining normal weight across adulthood, especially the prevention of weight gain in early adulthood, for reducing mortality risk in later life.

\section{Comparison with other studies}

The association between BMI and mortality has been extensively studied in many prospective cohort studies. However, most studies were conducted in middle aged or older participants and in general a $\mathrm{U}$ shaped association was reported. ${ }^{2}$ In our study, we reached a similar conclusion by using the baseline BMI as the exposure when the mean age of the participants was 57 years old. However, our study and several other studies have clearly shown that BMI in early adulthood is linearly associated with higher risk of mortality in later life. ${ }^{153132}$ In addition, we found that approximately $80 \%$ of the participants who were obese in young adulthood remained obese in middle or late adulthood (data not shown), so our results are also consistent with previous studies showing that longer duration of obesity was associated with higher mortality risk. ${ }^{1833}$ In addition to the obesity status at a single time point, more studies are now focusing on changes in body weight and health outcomes because weight changes are common across adulthood. Several studies with repeated measures of BMI have reported that both weight loss and weight gain in middle aged and older adults were associated with increased mortality risk, and a $45 \%$ and $7 \%$ higher risk of all cause mortality was reported in a meta-analysis of 26 cohort studies in middle aged and older adults. ${ }^{4}$ We also saw similar findings in our recent analysis in the Singapore Chinese population. ${ }^{34}$ In the study reported here, stable obesity and weight loss from an obese to a non-obese pattern from middle to late adulthood had a $20 \%$ and $30 \%$ higher mortality risk respectively. Extreme weight gain ( $\geq 20 \mathrm{~kg}$ ) in the 10 years before baseline was associated with a $36 \%$ higher mortality risk, whereas low to moderate weight gain (ranging from $2.5 \mathrm{~kg}$ to $20 \mathrm{~kg}$ ) or weight gain from non-obese to obese BMI was not significantly associated with mortality risk. Therefore, the results were overall consistent with the previous studies. ${ }^{4}$

Our study has the unique feature of examining the weight change patterns across adulthood and mortality risk in later life. We found that the relations of stable obesity and weight gain from non-obese to obese BMI with mortality were generally stronger from young to middle adulthood than from middle to late adulthood. Weight gain in adults mostly occurs from young to middle adulthood, but studies in this critical period are still lacking. ${ }^{35}$ A study in 1657 Finnish men with mean baseline age of 47 years reported that mortality risk was significantly increased in the highest quarter of weight gain $(\geq 15.0 \mathrm{~kg})$ from age 25 years to baseline compared with the lowest quarter. ${ }^{8}$ In the Southern Community Cohort study with 56868 US participants aged 40-65 at baseline, Hirko et al reported that being obese at 21 years old only or in both young and middle adulthood was significantly associated with higher mortality risk, but weight gain from non-obesity to obesity was not. ${ }^{15}$ The reasons for the inconsistent results might be that this study used stable non-obesity as the reference group and most of the participants were black Americans, in whom the authors previously reported that obesity in mid to late adulthood was not associated with higher mortality risk. ${ }^{36}$ Using data from the National Institutes of Health-AARP cohort with 109947 never smokers aged 50-71 years at entry, Adams et al found that weight gain was positively related to mortality, with stronger associations for weight gain from age 18 to 35 years and from 35 to 50 years than from age 50 to 69 years. ${ }^{10}$ The National Institutes of Health-AARP study also found that weight loss in late adulthood, but not 


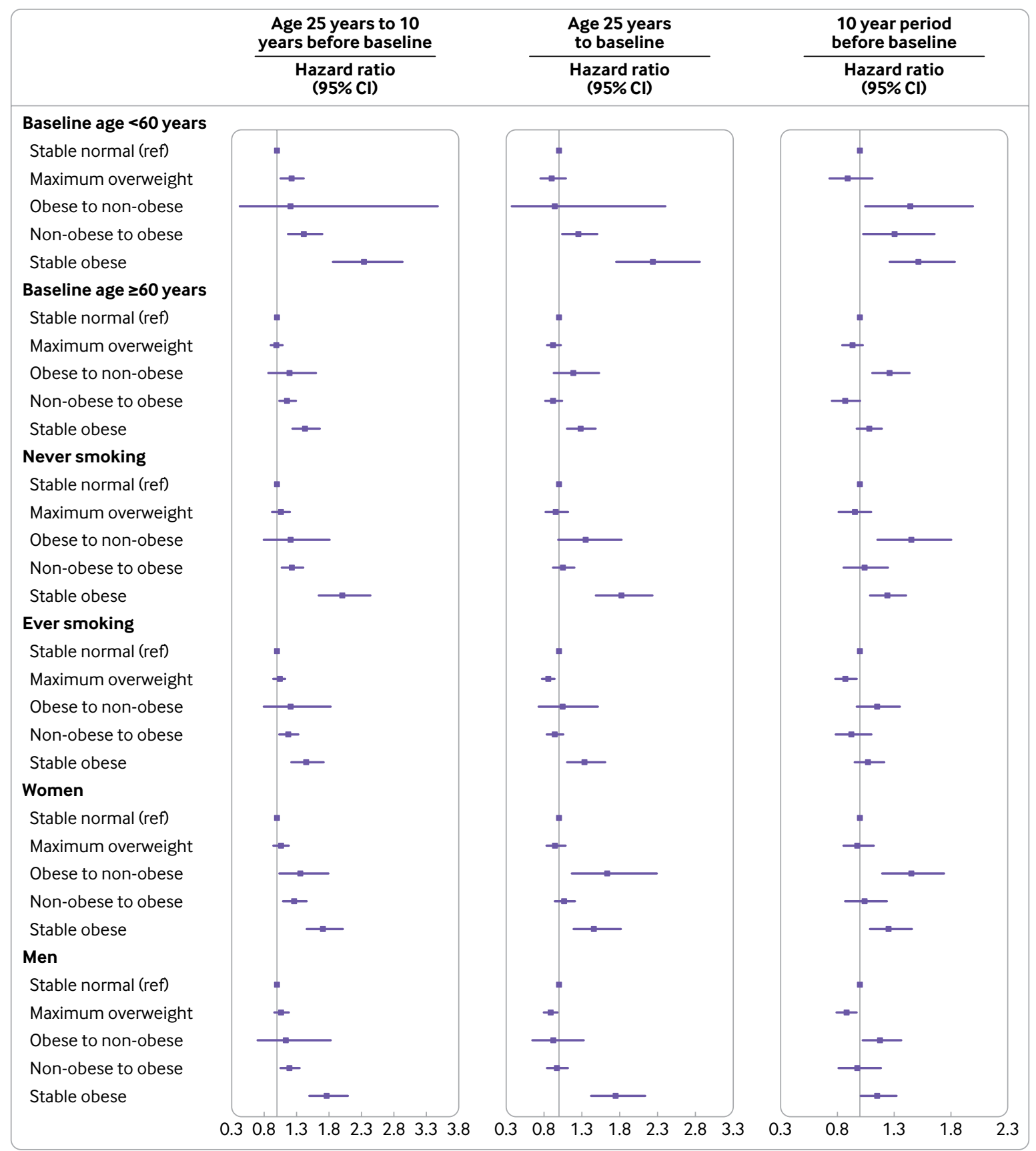

Fig 1 | Associations between weight change patterns across adulthood and risk of all cause mortality stratified by baseline age, smoking status, and sex in NHANES 1988-2014. Risk estimates were adjusted for baseline age (not adjusted in subgroup analysis by age), sex (not adjusted in subgroup analysis by sex), race/ethnicity, education level, family income-poverty ratio level, marital status, drinking status, smoking status (not adjusted in subgroup analysis by smoking status), leisure time physical activity level, healthy eating index scores, self reported general health, and family history of diabetes or heart attack. All estimates accounted for complex survey design of NHANES. In analysis of weight change from age 25 years to 10 years before baseline, $P$ for interaction was $<0.001$ for baseline age group ( 600 and $\geq 60$ years old), 0.97 for smoking status (never and ever smokers), and 0.84 for sex. In analysis of weight change from age 25 years to baseline, corresponding $P$ values for interaction were $<0.001,0.68$, and 0.86 . In analysis of weight change in 10 year period before baseline, corresponding $P$ values for interaction were $<0.001,0.44$, and 0.77

in early adulthood, was significantly associated with increased mortality risk. Our results are consistent with these studies and indicate that avoiding obesity at young age and preventing weight gain from young to middle adulthood could be an important strategy to reduce future mortality risk.

Several studies have also investigated the relation of weight change from young to late adulthood and mortality risk, but the results were not entirely consistent. Some studies found that weight gain over this period was associated with increased risk of all cause mortality, ${ }^{9-11}$ whereas other studies reported that weight loss, but not weight gain, was related to increased mortality risk, ${ }^{1237}$ which was similar to our results. The inconsistency might be due to the differences in sample size (ranging from 2512 to 118140 ) and substantial 

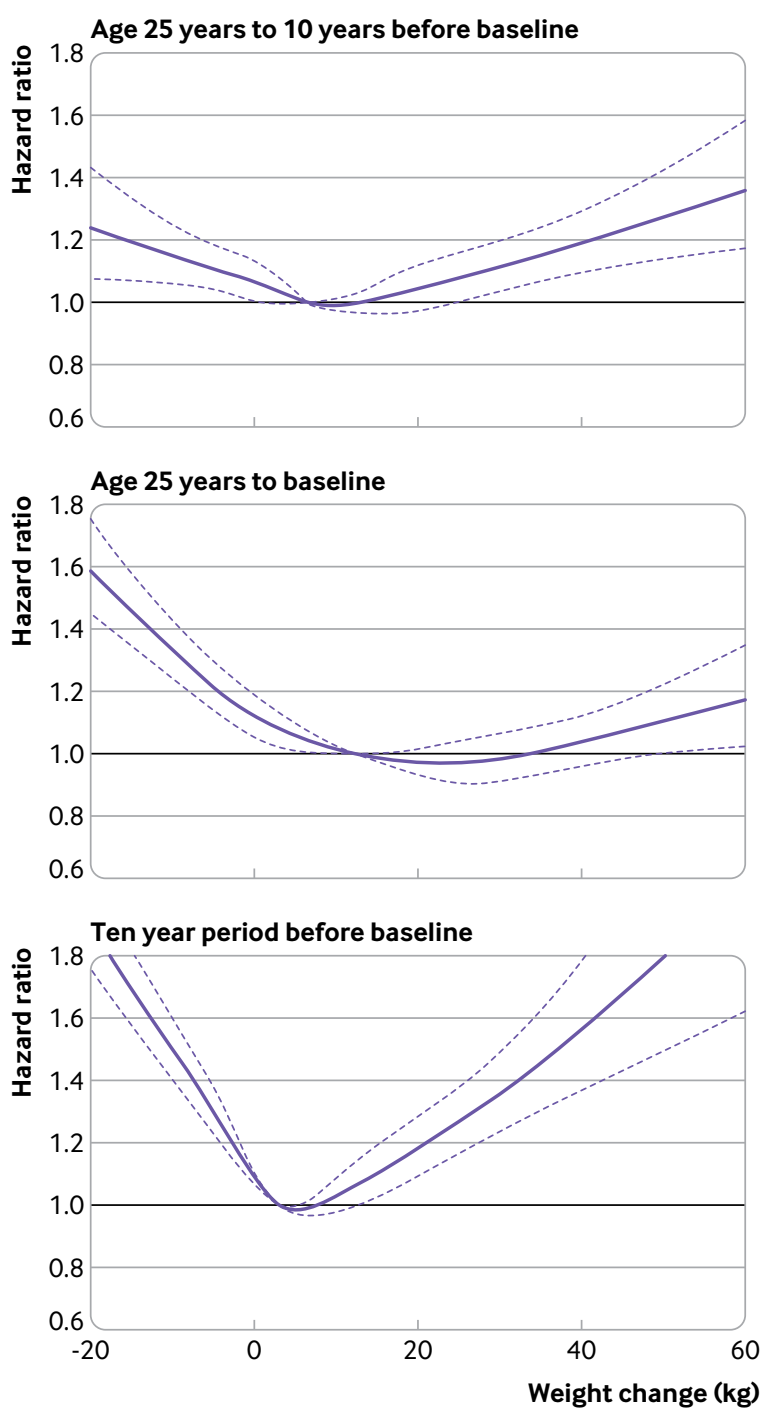

Fig 2 | Dose-response association between absolute weight change across adulthood and risk of all cause mortality. Associations were examined by multivariable Cox regression models based on restricted cubic splines. Solid line represents estimates of hazard ratios and dashed line represents $95 \% \mathrm{Cls}$. Risk estimates were adjusted for baseline age, sex, race/ethnicity, education level, family income-poverty ratio level, marital status, drinking and smoking status, leisure time physical activity level, healthy eating index scores, self reported general health, and family history of diabetes or heart attack. For weight change from age 25 years to 10 years before baseline or to baseline, weight at age 25 and baseline height were also adjusted for. For weight change from 10 years before baseline to baseline, weight at 10 years previously and baseline height were also adjusted for. $P$ values for overall association and $P$ values for non-linear association were all $<0.001$ in three periods

variations in the definitions of weight change across studies, which make direct comparisons difficult. In addition, the baseline age varied in different studies and previous studies showed that the obesity-mortality association tended to decline with increasing age. ${ }^{38} 39$ As also shown in our study, the association between weight gain and mortality attenuated as people got older, whereas the association with weight loss became stronger and significant in middle to late adulthood.

In this study, we found that both weight gain from non-obesity in young adulthood to obesity in middle adulthood and weight loss from obesity in middle adulthood to non-obesity in late adulthood were associated with higher risk of heart disease mortality. The results were consistent with some previous studies of cardiovascular disease mortality. ${ }^{11}{ }^{40}$ Weight change patterns were not significantly associated with cancer mortality across adulthood in our study, but we did not have data on incident cancer or site specific cancer mortality, so more studies are still needed to examine the relations between weight change and cancer risk. In our study, maximum overweight was generally not significantly related to mortality risk. This weight change pattern consisted of three heterogeneous groups, and the results should be interpreted cautiously. The positive relations of stable overweight and normal to overweight patterns with mortality were attenuated or even reversed with increasing age, which was consistent with our main recommendation of preventing weight gain at an early age. Weight loss from overweight to normal weight was consistently associated with higher risk of mortality across adulthood, and more studies are needed to confirm the results in other populations and explore the potential effect of weight loss among overweight people.

\section{Strengths and limitations of study}

This study had several strengths including its large and nationally representative sample, high follow-up rate, and detailed analysis of weight change from young adulthood through midlife to late adulthood. Our study shows the importance of examining the weight change patterns in different life periods given that people are more likely to gain weight and accumulate weight rapidly from young to middle adulthood, whereas weight begins to stabilise or even decrease from middle to late adulthood. ${ }^{6}$ Furthermore, weight gain from young to middle adulthood mostly reflects accumulation of fat mass, whereas weight loss in middle and late adulthood is usually accompanied by comorbidities and a decrease in lean mass and an increase in fat mass, ${ }^{78}$ so the underlying mechanisms of the harmful effect of weight change in certain life periods might differ. Another strength of our research is that we adjusted for a large number of potential confounders, including socioeconomic status and lifestyle factors. However, some factors, such as physical activity, diet quality, and self reported health status at baseline, could also be mediators for the relations of weight status and weight change patterns in young and middle adulthood with mortality risk, because they could be influenced by weight status and weight changes in young adulthood. ${ }^{41-43}$

Our study also has several limitations. Firstly, our approximation of middle adulthood (age range 30-80 years, interquartile range 36-56) overlapped with late adulthood (age range 40-90, interquartile range 46-66). However, in the analysis stratified by baseline age, the results remained and were even stronger in participants under 60 years old at baseline, which further confirmed that weight gain and stable obesity in younger adulthood were associated with higher mortality risk. Secondly, we used recalled and self reported weight data at age 25 years and 10 


\begin{tabular}{|c|c|c|c|c|c|}
\hline \multirow[b]{2}{*}{ All cause mortality } & \multicolumn{5}{|c|}{ Weight change in three life periods } \\
\hline & Weight loss $\geq 2.5 \mathrm{~kg}$ & $\begin{array}{l}\text { Weight change within } \\
2.5 \mathrm{~kg}\end{array}$ & $\begin{array}{l}\text { Weight gain } \geq 2.5 \mathrm{~kg} \text { and } \\
<10.0 \mathrm{~kg}\end{array}$ & $\begin{array}{l}\text { Weight gain } \geq 10 \mathrm{~kg} \text { and } \\
<20 \mathrm{~kg}\end{array}$ & Weight gain $\geq 20 \mathrm{~kg}$ \\
\hline \multicolumn{6}{|c|}{ From age 25 years to 10 years before baseline } \\
\hline No of deaths/person years & $755 / 18364$ & $2146 / 83396$ & $2643 / 108375$ & $2174 / 76212$ & $1680 / 47628$ \\
\hline Age adjusted mortality rate† & 36.9 (34.3 to 39.6$)$ & $30.0(28.7$ to 31.3$)$ & 26.1 (25.1 to 27.1$)$ & $26.0(24.9$ to 27.1$)$ & $31.0(29.4$ to 32.5$)$ \\
\hline Model 1 & $1.30(1.10$ to 1.53$)$ & 1.00 & $0.90(0.82$ to 0.99$)$ & 0.95 (0.86 to 1.05$)$ & $1.16(1.04$ to 1.30$)$ \\
\hline Model 2 & $1.08(0.92$ to 1.27$)$ & 1.00 & $0.97(0.87$ to 1.07$)$ & 1.05 (0.94 to 1.17$)$ & $1.28(1.15$ to 1.42$)$ \\
\hline Model 3 & $1.10(0.94$ to 1.28$)$ & 1.00 & $0.96(0.88$ to 1.06$)$ & 1.01 (0.91 to 1.12$)$ & $1.19(1.07$ to 1.32$)$ \\
\hline \multicolumn{6}{|l|}{ From age 25 years to baseline } \\
\hline No of deaths/person years & $1801 / 32451$ & $1059 / 31534$ & $2027 / 76207$ & $2417 / 98014$ & $2277 / 100197$ \\
\hline Age adjusted mortality rate $\dagger$ & $44.4(42.2$ to 46.6$)$ & 29.1 (27.3 to 30.9$)$ & $26.5(25.4$ to 27.7$)$ & $25.6(24.6$ to 26.6$)$ & $25.7(24.5$ to 26.8$)$ \\
\hline Model 1 & $1.44(1.26$ to 1.64$)$ & 1.00 & 0.97 (0.86 to 1.09$)$ & 0.91 (0.82 to 1.02$)$ & 1.09 (0.98 to 1.21$)$ \\
\hline Model 2 & 1.20 (1.04 to 1.38$)$ & 1.00 & $1.00(0.89$ to 1.12$)$ & 0.99 (0.89 to 1.10$)$ & $1.14(1.03$ to 1.27$)$ \\
\hline Model 3 & $1.19(1.04$ to 1.35$)$ & 1.00 & $0.99(0.90$ to 1.10$)$ & $0.96(0.86$ to 1.06$)$ & $1.04(0.94$ to 1.15$)$ \\
\hline \multicolumn{6}{|c|}{ From 10 years before baseline to baseline } \\
\hline No of deaths/person years & $3853 / 77387$ & $2205 / 72259$ & $2184 / 101632$ & $1115 / 62767$ & $570 / 31138$ \\
\hline Age adjusted mortality ratet & $38.5(37.2$ to 39.7$)$ & 25.5 (24.4 to 26.6$)$ & $23.6(22.6$ to 24.6$)$ & $25.4(23.7$ to 27.1$)$ & $28.5(25.3$ to 31.6$)$ \\
\hline Model 1 & $1.49(1.35$ to 1.64$)$ & 1.00 & $0.99(0.91$ to 1.07$)$ & 1.14 (1.04 to 1.25$)$ & $1.68(1.43$ to 1.97$)$ \\
\hline Model 2 & 1.29 (1.16 to 1.42$)$ & 1.00 & $1.00(0.92$ to 1.08$)$ & 1.11 (1.01 to 1.22$)$ & 1.54 (1.31 to 1.82$)$ \\
\hline Model 3 & 1.26 (1.14 to 1.39$)$ & 1.00 & $0.99(0.91$ to 1.07$)$ & $1.04(0.94$ to 1.15$)$ & $1.36(1.17$ to 1.57$)$ \\
\hline \multicolumn{6}{|c|}{$\begin{array}{l}\text { *All estimates accounted for complex survey designs. Among } 36051 \text { participants, a total of } 2751,2271 \text {, and } 1469 \text { participants were excluded in analyses from age } 25 \text { years to } 10 \text { years before } \\
\text { baseline, age } 25 \text { years to baseline, and } 10 \text { years before baseline to baseline, respectively, owing to missing values of body mass index at both times. Model } 1 \text { was adjusted for baseline age, sex } \\
\text { race/ethnicity. Mode } 2 \text { was additionally adjusted for education level, family income-poverty ratio level, marital status, drinking and smoking status, and family history of diabetes or heart attack. } \\
\text { For weight change from age } 25 \text { years to } 10 \text { years before baseline or to baseline, weight at age } 25 \text { and baseline height were also adjusted in model } 2 \text {. For weight change from } 10 \text { years before } \\
\text { baseline to baseline, weight at } 10 \text { years ago and baseline height were also adjusted in model } 2 \text {. Model } 3 \text { was further adjusted for baseline leisure time physical activity level, healthy eating inde } \\
\text { scores, and self reported general health. } \\
\text { tMortality rates per } 1000 \text { person years, directly standardised to age distribution of entire study population. }\end{array}$} \\
\hline
\end{tabular}

years before the baseline NHANES survey and thus misclassification bias may have been introduced. However, several validation studies and a recent metaanalysis showed that recalled early life weight could be a valid measure to use in life course epidemiological analysis. ${ }^{44}$ Thirdly, many covariates were available only at baseline, so we could not use time varying covariates to capture changes in possible confounders over time. Fourthly, we could not differentiate intentional from unintentional weight change, and additional information on reasons of weight change may enable better interpretation of the results of future studies and thus appropriate intervention strategies. Lastly, we did not evaluate the relations of changes in other adiposity related markers such as waist circumference and fat mass with mortality owing to lack of data at different time points. Further studies with repeated data on these markers may provide a more comprehensive picture of the changes in obesity status and mortality risk.

\section{Conclusions and public health implications}

Our study found that stable obesity across adulthood and weight gain from young to middle adulthood was associated with increased risks of all cause and heart disease mortality, as was weight loss in middle to late adulthood. Future studies are needed to unravel the mechanisms underlying the association between weight change across adulthood and mortality, particularly the relations of changes in body composition to mortality. In addition, as weight loss is less achievable (only 1.4\% participants changed from the obese to the non-obese category from young to late adulthood), our results suggest that prevention of weight gain might be more important. Taken together, the findings indicate that maintaining normal weight throughout the adulthood, especially prevention of weight gain in early adulthood, should be encouraged to reduce risk of premature deaths. Finally, further studies are still needed to consider other health related and obesity related outcomes, and clinical trials on the long term health consequences of weight loss are also warranted.

Contributors: CC, XFP, and AP conceived the study design. CC and YY did the statistical analysis. CC and AP wrote the manuscript. All authors provided critical revisions of the draft and approved the submitted draft. The corresponding author attests that all listed authors meet authorship criteria and that no others meeting the criteria have been omitted. AP is the guarantor.

Funding: This study was supported by grants from the National Key Research and Development Program of China to AP (2017YFC0907504) and the China Postdoctoral Science Foundation (No 176596) and International Postdoctoral Exchange Fellowship of the China Postdoctoral Council (No 20180062) to XFP. The funders had no role in design and conduct of the study; collection, management, analysis, and interpretation of the data; preparation, review, and approval of the manuscript; or the decision to submit the manuscript for publication

Competing interests: All authors have completed the ICMIE uniform disclosure form at http://www.icmje.org/coi_disclosure.pdf (available on request from the corresponding author) and declare: no support from any organisation for the submitted work other than those described above; no financial relationships with any organisations that might have an interest in the submitted work in the previous three years; no other relationships or activities that could appear to have influenced the submitted work.

Ethical approval: Approval of the study from the National Center of Health and Statistics Research ethics review board was waived because the research relied on publicly used, de-identified secondary data.

Transparency declaration: The manuscript's guarantor affirms that the manuscript is an honest, accurate, and transparent account of the study being reported; that no important aspects of the study have been omitted; and that any discrepancies from the study as planned (and, if relevant, registered) have been explained.

Data sharing: No additional data available.

This is an Open Access article distributed in accordance with the Creative Commons Attribution Non Commercial (CC BY-NC 4.0) license, 
which permits others to distribute, remix, adapt, build upon this work non-commercially, and license their derivative works on different terms, provided the original work is properly cited and the use is noncommercial. See: http://creativecommons.org/licenses/by-nc/4.0/.

1 Abarca-Gómez L, Abdeen ZA, Hamid ZA, et al, NCD Risk Factor Collaboration (NCD-RisC). Worldwide trends in body-mass index, underweight, overweight, and obesity from 1975 to 2016: a pooled analysis of 2416 population-based measurement studies in 128.9 million children, adolescents, and adults. Lancet 2017;390:262742. doi:10.1016/S0140-6736(17)32129-3

2 Di Angelantonio E, Bhupathiraju ShN, Wormser D, et al, Global BMI Mortality Collaboration. Body-mass index and all-cause mortality: individual-participant-data meta-analysis of 239 prospective studies in four continents. Lancet 2016;388:776-86. doi:10.1016/S01406736(16)30175-1

3 Bhaskaran K, Dos-Santos-Silva I, Leon DA, Douglas IJ, Smeeth L. Association of BMI with overall and cause-specific mortality: a population-based cohort study of 3.6 million adults in the UK. Lancet Diabetes Endocrinol 2018;6:944-53. doi:10.1016/S22138587(18)30288-2

4 Karahalios A, English DR, Simpson JA. Change in body size and mortality: a systematic review and meta-analysis. Int J Epidemiol 2017;46:526-46.

5 Cheng FW, Gao X, Jensen GL. Weight change and all-cause mortality in older adults: a meta-analysis. J Nutr Gerontol Geriatr 2015;34:343-68. doi:10.1080/21551197.2015.1090362

6 Sheehan TJ, DuBrava S, DeChello LM, Fang Z. Rates of weight change for black and white Americans over a twenty year period. Int J Obes Relat Metab Disord 2003;27:498-504. doi:10.1038/ sj.ijo.0802263

7 Fontana L, Hu FB. Optimal body weight for health and longevity: bridging basic, clinical, and population research. Aging Cell 2014;13:391-400. doi:10.1111/acel.12207

8 Strandberg TE, Strandberg A, Salomaa VV, Pitkälä K, Miettinen TA. Impact of midlife weight change on mortality and quality of life in old age. Prospective cohort study. Int J Obes Relat Metab Disord 2003;27:950-4. doi:10.1038/sj.ijo.0802313

9 Shimazu T, Kuriyama S, Ohmori-Matsuda K, Kikuchi N, Nakaya N, Tsuji I. Increase in body mass index category since age 20 years and all-cause mortality: a prospective cohort study (the Ohsaki Study). Int J Obes (Lond) 2009;33:490-6. doi:10.1038/ijo.2009.29

10 Adams KF, Leitzmann MF, Ballard-Barbash R, et al. Body mass and weight change in adults in relation to mortality risk. Am J Epidemiol 2014;179:135-44. doi:10.1093/aje/kwt254

11 Zheng Y, Manson JE, Yuan C, et al. Associations of Weight Gain From Early to Middle Adulthood With Major Health Outcomes Later in Life. JAMA 2017;318:255-69. doi:10.1001/jama.2017.7092

12 Yarnell JW, Patterson CC, Thomas HF, Sweetnam PM. Comparison of weight in middle age, weight at 18 years, and weight change between, in predicting subsequent 14 year mortality and coronary events: Caerphilly Prospective Study. J Epidemiol Community Health 2000:54:344-8. doi:10.1136/jech.54.5.344

13 Jeffreys M, McCarron P, Gunnell D, McEwen J, Smith GD. Body mass index in early and mid-adulthood, and subsequent mortality: a historical cohort study. Int J Obes Relat Metab Disord 2003;27:13917. doi:10.1038/sj.ijo.0802414

14 Corrada MM, Kawas CH, Mozaffar F, Paganini-Hill A. Association of body mass index and weight change with all-cause mortality in the elderly. Am J Epidemiol 2006;163:938-49. doi:10.1093/aje/kwj114

15 Hirko KA, Kantor ED, Cohen SS, Blot WJ, Stampfer MJ, Signorello LB. Body mass index in young adulthood, obesity trajectory, and premature mortality. Am J Epidemiol 2015;182:441-50. doi:10.1093/aje/kwv084

16 Mehta NK, Stenholm S, Elo IT, Aromaa A, Heliövaara M, Koskinen S. Weight histories and mortality among finnish adults: the role of duration and peak body mass index. Epidemiology 2014;25:707-10. doi:10.1097/EDE.0000000000000147

17 Song M, Hu FB, Wu K, et al. Trajectory of body shape in early and middle life and all cause and cause specific mortality: results from two prospective US cohort studies. BMJ 2016;353:i2195. doi: $10.1136 /$ bmj.i2195

18 Preston SH, Mehta NK, Stokes A. Modeling obesity histories in cohort analyses of health and mortality. Epidemiology 2013;24:15866. doi:10.1097/EDE.0b013e3182770217

19 Centers for Disease Control and Prevention (CDC). About the National Health and Nutrition Examination Survey. 2017 https://www.cdc.gov/ nchs/nhanes/about_nhanes.htm.

20 NHLBI Obesity Education Initiative Expert Panel on the Identification, Evaluation, and Treatment of Obesity in Adults. Clinical Guidelines on the Identification, Evaluation, and Treatment of Overweight and Obesity in Adults. Report no. 98-4083. National Heart, Lung, and Blood Institute, 1998.
21 Stokes A, Collins JM, Grant BF, et al. Obesity progression between young adulthood and midlife and incident diabetes: a retrospective cohort study of U.S. adults. Diabetes Care 2018;41:1025-31. doi: $10.2337 /$ dc $17-2336$

22 National Center for Health Statistics. 2015 Public-Use Linked Mortality Files. 2019. https://www.cdc.gov/nchs/data-linkage/ mortality-public.htm\#.

23 Rao JN, Scott AJ. On chi-squared tests for multiway contingency tables with cell proportions estimated from survey data. Ann Stat 1984:46-60. doi:10.1214/aos/1176346391

24 Berrington de Gonzalez A, Hartge P, Cerhan JR, et al. Body-mass index and mortality among 1.46 million white adults. N Englf Med 2010;363:2211-9. doi:10.1056/NEJMoa1000367

25 Durrleman S, Simon R. Flexible regression models with cubic splines. Stat Med 1989;8:551-61. doi:10.1002/sim.4780080504

26 Brown AF, Liang L-J, Vassar SD, et al. Trends in racial/ethnic and nativity disparities in cardiovascular health among adults without prevalent cardiovascular disease in the United States, 1988 to 2014. Ann Intern Med 2018;168:541-9. doi:10.7326/M17-0996

27 Chen F, Du M, Blumberg JB, et al. Association Among Dietary Supplement Use, Nutrient Intake, and Mortality Among U.S. Adults: A Cohort Study. Ann Intern Med 2019;170:604-13. doi:10.7326/M182478

28 Piercy KL, Troiano RP, Ballard RM, et al. The physical activity guidelines for Americans. JAMA 2018;320:2020-8. doi:10.1001/ jama.2018.14854

29 National Cancer Institute. Developing the Healthy Eating Index. 2019. https://epi.grants.cancer.gov/hei/developing.html\#f1b.

30 Yuan Y. Multiple imputation using SAS software. / Stat Softw 2011:45:1-25. doi:10.18637/iss.v045.i06

31 Ma J, Flanders WD, Ward EM, Jemal A. Body mass index in young adulthood and premature death: analyses of the US National Health Interview Survey linked mortality files. Am J Epidemiol 2011;174:934-44. doi:10.1093/aje/kwr169

32 Stevens J, Truesdale KP, Wang CH, Cai J, Erber E. Body mass index at age 25 and all-cause mortality in whites and African Americans: the Atherosclerosis Risk in Communities study. J Adolesc Health 2012;50:221-7. doi:10.1016/j.jadohealth.2011.06.006

33 Abdullah A, Wolfe R, Stoelwinder JU, et al. The number of years lived with obesity and the risk of all-cause and cause-specific mortality. Int J Epidemiol 2011;40:985-96. doi:10.1093/ije/dyr018

34 Pan XF, Yuan JM, Koh WP, Pan A. Weight change in relation to mortality in middle-aged and elderly Chinese: the Singapore Chinese Health Study. Int J Obes (Lond) 2019;43:1590-600. doi:10.1038/ s41366-018-0259-y

35 Dietz WH. Obesity and excessive weight gain in young adults: new targets for prevention. JAMA 2017;318:241-2. doi:10.1001/ jama.2017.6119

36 Cohen SS, Signorello LB, Cope EL, et al. Obesity and allcause mortality among black adults and white adults. Am J Epidemiol 2012;176:431-42. doi:10.1093/aje/kws032

37 Drøyvold WB, Lund Nilsen TI, Lydersen S, et al, Nord-Trøndelag Health Study. Weight change and mortality: the Nord-Trøndelag Health Study. J Intern Med 2005;257:338-45. doi:10.1111/j.13652796.2005.01458.x

38 Ferrucci L, Alley D. Obesity, disability, and mortality: a puzzling link. Arch Intern Med 2007;167:750-1. doi:10.1001/archinte.167.8.750

39 Elliott AM, Aucott LS, Hannaford PC, Smith WC. Weight change in adult life and health outcomes. Obes Res 2005;13:1784-92. doi:10.1038/oby.2005.217

40 Park SY, Wilkens LR, Maskarinec G, Haiman CA, Kolonel LN, Marchand LL. Weight change in older adults and mortality: the Multiethnic Cohort Study. Int J Obes (Lond) 2018;42:205-12. doi:10.1038/ijo.2017.188

41 Ritchie LD, Spector P, Stevens MJ, et al. Dietary patterns in adolescence are related to adiposity in young adulthood in black and white females. J Nutr 2007;137:399-406. doi:10.1093/ jn/137.2.399

42 Hulman A, Ibsen DB, Laursen ASD, Dahm CC. Body mass index trajectories preceding first report of poor self-rated health: $A$ longitudinal case-control analysis of the English Longitudinal Study of Ageing. PLoS One 2019;14:e0212862. doi:10.1371/journal. pone.0212862

43 Golubic R, Ekelund U, Wijndaele K, et al. Rate of weight gain predicts change in physical activity levels: a longitudinal analysis of the EPIC-Norfolk cohort. Int I Obes (Lond) 2013;37:404-9. doi:10.1038/ijo.2012.58

44 De Rubeis V, Bayat S, Griffith LE, Smith BT, Anderson LN. Validity of self-reported recall of anthropometric measures in early life: A systematic review and meta-analysis. Obes Rev 2019;20:1426-40. doi:10.1111/obr.12881

Supplementary materials 